\title{
Development and feasibility of an eHealth tool for idiopathic pulmonary fibrosis
}

\begin{abstract}
To the Editor:
Idiopathic pulmonary fibrosis (IPF) is a chronic, progressive disease with a poor prognosis. The quality of life of patients is often impaired $[1,2]$. In other chronic lung diseases, the use of eHealth to improve clinical outcomes has been increasingly investigated [3-5]. eHealth is defined as "the use of information and communication technologies for health" [6]. Use of eHealth may improve understanding of disease, promote self-management and facilitate longitudinal data collection for both care and research [3, 7]. Experience with eHealth tools in IPF is scarce and people are often hesitant to start online initiatives in this mostly elderly population. However, collecting data at home and facilitating consultations at a distance could hold great benefits for these patients, as they often struggle to visit hospital because of reduced mobility, dyspnoea and extra oxygen needs. Together with patients we developed IPF-online, an eHealth tool for patients with IPF, and evaluated the feasibility and user satisfaction of this tool.

During two pulmonary fibrosis information meetings in 2014 and 2015 at our hospital, patients were asked whether they would like to keep track of their disease online; $82 \%$ of patients $(n=67)$ responded with "yes". In response to this, we developed an eHealth tool, based on available information from literature, experiences in other fields and individual patient suggestions. This resulted in IPF-online (www.ipfonline.nl), a secured personal platform which contains information about IPF, patient-reported outcome measures (PROMs), medication use and an eConsult possibility. Results of hospital-based lung function measurements are imported by the healthcare provider. Patients remain owner of their data, and give digital informed consent for clinical or research purposes. Data is stored in high-end ISO 27001 certified data centres. Patients access IPF-online via personal codes, in compliance with European safety regulations.
\end{abstract}

The prototype of IPF-online was submitted to the medical ethical committee and approval was granted to further develop and evaluate the tool together with patients in a hands-on manner.

Two consecutive groups of outpatients, with a diagnosis of IPF [1], were invited to participate. Patients were given access to their personal platform and were asked to report medication use and PROMs at baseline and after 14 days in IPF-online. Symptoms as cough, fatigue and breathlessness were assessed on a visual analogue scale. Patients also completed different questionnaires, such as the King's Brief Interstitial Lung Disease health status and the Euroqol 5D-5L $[8,9]$. An evaluation questionnaire was sent afterwards to assess patient experiences. We used suggestions of patients to further develop and improve IPF-online. Subsequently, a second group of patients was asked to test and evaluate the adapted version of IPF-online.

In total, 27 patients participated; 18 patients in the first group, and nine patients in the second group. The mean age was 67 years (range 56-86 years); most patients were male (85\%); median forced vital capacity was $78 \%$ of predicted (range $46-131 \%$ ) and mean transfer factor for carbon monoxide corrected for haemoglobin was $50 \%$ (range 16-79\%).

All patients managed to use IPF-online and complete electronic PROMs without help from healthcare providers. Patients provided constructive feedback on IPF-online and suggested different features that could be added to improve the tool, such as the choice of tables and graphs to display longitudinal data. The first group suggested adding educational movies about IPF and information about medication and side-effects. These patient recommendations were used to adapt IPF-online. The second group additionally 
mentioned the need for a better explanation about PROM scores and video consultation with the healthcare provider, which will be implemented.

In both groups, many patients used the information platform (on average twice a week per patient) and the eConsult option (table 1). More than two-thirds of patients considered IPF-online easy to use, which increased after the adaptations made according to the suggestions of the first group. The vast majority had positive experiences (table 1). Some patients mentioned the advantages of the interactive part of IPF-online: "I like it because you can have easier communication with doctors and nurses", "clear questions and useful interaction with health carers", "the eConsult option is very useful", and "I am immediately updated".

Other patients commented on the information platform: "IPF-online makes it possible to absorb information at my own pace", and "all useful information is gathered in one place".

Almost all patients wished to continue using IPF-online: "the more contact you have, the better it is", "I like having my own follow-up data", and "it gives a good overview".

A few patients (3/27) were less satisfied: "completing online questionnaires is too time consuming", "an internet tool does not have added value for me", and "I can't find all available information".

This pilot study shows that the use of an eHealth tool is feasible in patients with IPF. Patient satisfaction was high and most patients continued the use of IPF-online.

One of the factors that may account for the high patient satisfaction is the multi-step co-development approach we took to create IPF-online. Previous research has shown that perspectives of healthcare providers and patients regarding eHealth might differ [3]. In our project ease to use improved after patients recommended changes, underlining the importance of patient contribution.

The age range of our cohort was 56-86 years. As IPF occurs mostly in an elderly population, internet access and experience with online tools may be questioned. However, European data show that internet use is steadily increasing among people aged over 65 years [10]. Furthermore, studies in chronic obstructive pulmonary disease with a similar age range, showed that eHealth technologies were feasible in this population $[5,11]$.

eHealth solutions have the potential to improve care and facilitate research for patients with IPF. We believe that eHealth may enable earlier identification of inter-current problems and disease deterioration. Need for more and adequate information, and shared decision-making is repeatedly reported in IPF [12, 13]. IPF-online provides patients with more insight into their own disease, can guide personalised treatment decisions and can be used as outcome parameter for both research and clinical practice. Expansion with home spirometry is currently being investigated.

In the current study, we obtained 100\% PROMs completion rate, which may have definite benefits for research, avoiding missing data. This is in line with the opinion of the ePRO task force of the International Society for Pharmacoeconomics and Outcomes Research, stating that electronic PROMs have advantages over paper-based questionnaires and provide equivalent, reliable outcomes [14, 15]. The questionnaires incorporated in IPF-online were not validated for online use. However, current evidence shows that full psychometric validation of ePROMs is not necessary when only minor modifications are made to the original PROMs [14]. Testing usability of ePROMs in a small group, as done in the current

TABLE 1 Patient experiences and use of IPF-online during 14 days pilot study

Group $1(n=18)$

Group 2 (n=9)

\section{Use of IPF-online}

Completion of PROMs

Information platform

$100 \%-100 \%$

eConsult

$100 \%$

$100 \%$

Patient experiences

$33 \%$

$44 \%$

Easy to use

Useful

$78 \%$

$89 \%$

Would recommend it to others

$89 \%$

$94 \%$

Wish to continue

$72 \%$

\section{$89 \%$}

$89 \%$

$89 \%$

$100 \%$

Spontaneously continued use of IPF-online after pilot

$100 \%$

PROMs: patient-reported outcome measures. 
study, to evaluate whether participants are able to use the device and software to complete the questionnaires is sufficient [14].

A limitation of this study is that it was a short pilot study in a relatively small and possibly more motivated patient group. This may be a reason for the 100\% PROM completion rate. Nonetheless, $82 \%$ of the overall patient population was motivated to use the eHealth tool. Furthermore, the group included a broad range in age and severity of disease and the majority of patients continued using the tool.

All together, we believe that the use of IPF-online is feasible and highly valued by patients with IPF. Whether its long-term use improves quality of life, medication use and end-point assessment for trials is a field for further studies.

Catharina C. Moor ${ }^{1}$, Mirjam J.G. van Manen ${ }^{1}$, Nelleke C. Tak ${ }^{1}$, Esther van Noort $^{2}$ and Marlies S. Wijsenbeek ${ }^{1}$

${ }^{1}$ Dept of Respiratory Medicine, Erasmus Medical Center, University Hospital Rotterdam, Rotterdam, The Netherlands. ${ }^{2}$ Curavista bv, Geertruidenberg, The Netherlands.

Correspondence: Marlies S. Wijsenbeek, Erasmus MC, University Hospital Rotterdam, Dept of Pulmonary Disease, 's-Gravendijkwal 230, Rotterdam 3015 CA, The Netherlands. E-mail: m.wijsenbeek-lourens@erasmusmc.nl

Received: Dec 042017 | Accepted after revision: Jan 062018

Conflict of interest: M.S. Wijsenbeek has received grants and fees (paid directly to the institution) from Hoffman-la Roche and Boehringer-Ingelheim, and fees (paid directly to the institution) from Galapagos, outside the submitted work.

\section{References}

1 Raghu G, Collard HR, Egan JJ, et al. An official ATS/ERS/JRS/ALAT statement: idiopathic pulmonary fibrosis: evidence-based guidelines for diagnosis and management. Am J Respir Crit Care Med 2011; 183: 788-824.

2 Yount SE, Beaumont JL, Chen SY, et al. Health-related quality of life in patients with idiopathic pulmonary fibrosis. Lung 2016; 194: 227-234.

3 Simpson AJ, Honkoop PJ, Kennington E, et al. Perspectives of patients and healthcare professionals on mHealth for asthma self-management. Eur Respir J 2017; 49: 1601966.

4 Gregersen TL, Green A, Frausing E, et al. Do telemedical interventions improve quality of life in patients with COPD? A systematic review. Int J Chron Obstruct Pulmon Dis 2016; 11: 809-822.

5 Hardinge M, Rutter $\mathrm{H}$, Velardo $\mathrm{C}$, et al. Using a mobile health application to support self-management in chronic obstructive pulmonary disease: a six-month cohort study. BMC Med Inform Decis Mak 2015; 15: 46.

World Health Organization. eHealth. www.who.int/ehealth/en/ Last accessed August 2017.

Wicks P, Stamford J, Grootenhuis MA, et al. Innovations in e-health. Qual Life Res 2014; 23: 195-203.

Patel AS, Siegert RJ, Brignall K, et al. The development and validation of the King's Brief Interstitial Lung Disease (K-BILD) health status questionnaire. Thorax 2012; 67: 804-810.

9 Brooks R. EuroQol: the current state of play. Health Policy 1996; 37: 53-72.

10 Eurostat. Eurostat Internet access and use statistics: households and individuals. http://ec.europa.eu/eurostat/ statistics-explained/index.php/Internet_access_and_use_statistics_households_and_individuals Date last accessed: November 13, 2017. Date last updated: January 30, 2017.

11 Sund ZM, Powell T, Greenwood R, et al. Remote daily real-time monitoring in patients with COPD: a feasibility study using a novel device. Respir Med 2009; 103: 1320-1328.

12 Bonella F, Wijsenbeek M, Molina-Molina M, et al. European IPF Patient Charter: unmet needs and a call to action for healthcare policymakers. Eur Respir J 2016; 47: 597-606.

13 Sampson C, Gill BH, Harrison NK, et al. The care needs of patients with idiopathic pulmonary fibrosis and their carers (CaNoPy): results of a qualitative study. BMC Pulm Med 2015; 15: 155.

14 Coons SJ, Gwaltney CJ, Hays RD, et al. ecommendations on evidence needed to support measurement equivalence between electronic and paper-based patient-reported outcome (PRO) measures: ISPOR ePRO Good Research Practices Task Force report. Value Health 2009; 12: 419-429.

15 Rutherford C, Costa D, Mercieca-Bebber R, et al. Mode of administration does not cause bias in patient-reported outcome results: a meta-analysis. Qual Life Res 2016; 25: 559-574. 